\title{
Inhibitory Potential of Rose Petals Extracts: An Attempt to Annihilate Staphylococcus aureus
}

\author{
C.J. Chandekar and Pranita A. Gulhane*
}

Department of Microbiology, S.S.E.S.A's Science College, Nagpur (MS), India

*Corresponding author

\section{A B S T R A C T}

\section{Keywords}

Rose petals, Staphylococcus aureus, Antibacterial activity

Article Info

Accepted:

12 November 2018 Available Online:

10 December 2018
Rose has influenced cultures artistically, economically, clinically, scientifically, psychologically and religiously because of its fragrance. The floral petals of higher plants are known to possess antibacterial activity. In the present study antimicrobial activity of rose petals extracts were evaluated against five strains of skin infection causing S. aureus. Antibiotic susceptibility test showed that $100 \% \mathrm{~S}$. aureus strains were found to be sensitive to Erythromycin and Linezolid each, followed by $80 \%$ to Clindamycin and $60 \%$ to Gatifloxacin. However, all the strains of $S$. aureus were resistant to Oxacillin. It was found that both the types of extracts prepared in methanol and distilled water were found to be effective against it.

\section{Introduction}

From ancient times, humans have utilized plants for the treatment or prevention of diseases, leading to the dawn of traditional medicine. While plant leaf, stem and root extracts have been widely evaluated for bioactive compounds, screening for plant flower has not been extensive. The flower petals which provide physical protection to the reproductive compounds can be expected to synthesize potent bioactive compounds. The floral petals of higher plants are known to possess antibacterial activity (Darokar et al., 1998). Roses have influenced cultures aesthetically, economically, medically, religiously and spiritually since humankind could smell and appreciate its fragrance. Rose is a perennial plant of the genus Rosa, within the family Rosaceae. Rose has influenced cultures artistically, economically, clinically, scientifically, psychologically and religiously because of its fragrance. There are approximately 10,000 species of rose; $R$. damascene, $R$. gallica, $R$. centifolia, $R$. indica, $R$. rubiginosa to name a few (Ozkan et al., 2004).

Rose is the most celebrated of all the fragrances reason being the presence of anthocyanins in the petals. Rose petals and leaves work as a diuretic and flush toxins from 
the body. They are known to relieve bronchial and chest congestion and also provide relief from sore throat. However, Staphylococcus aureus is a gram positive bacterium that usually appears under the microscope as spherical (coccus) organisms appearing in pairs, short chains, or bunched, grape-like clusters. Staphylococcus aureus has been implicated as a causative agent in acute food poisoning episodes, toxic shock syndrome, impetigo, scalded skin syndrome, cellulitis, folliculitis and furuncles. It is also a common cause of systemic infections such as infective endocarditis, osteomyelitis, epiglottitis, and sinus infections amongst others. S. aureus is also responsible for many infective and systemic infections in the health care setting (nosocomial infections). Rose water because of its antiseptic property has been used as eyewash, and is also used to moisturize the skin. Various studies have been conducted to establish the anti-fungal, antibacterial and anti-oxidant activities of rose. These activities were attributed to the presence of flavonoid and phenolic compounds, also known as bioactive agents (Kiruthika et al., 2011; Sahoo et al., 2011; Khan and Tewari, 2011; Saeed et al., 2014). Therefore the present study was conducted to evaluate the antibacterial property of Roses extract against Staphylococcus aureus.

\section{Materials and Methods}

\section{Sample collection}

The flowers were picked in morning and stored in at low temperature for further use. The petals were surface sterilized with $0.1 \%$ Mercuric Chloride for 10 seconds and washed with sterile distilled water for three successive times (Laxmi et al., 2017).

\section{Preparation of extract of rose petals}

From rose, the petals were removed and subjected to air drying under shade for one week. After drying completely it was grinded to make powder of it. A 5 gm of each samples were dissolved in $50 \mathrm{ml}$ of solvents i.e. in Methanol and Distilled water and allowed to stand for one week with frequent shaking. After one week the solvents were filtered through Whatman Filter Paper No. 1 and kept it in incubator at $37^{\circ} \mathrm{C}$ till all solvents had completely evaporated from mixtures. Now all mixtures were dissolved in Tris HCL (Cantore et al., 2009).

\section{Test organisms}

Skin infection causing Staphylococcus aureus were collected from pathology laboratory in Nagpur and were identified on the basis of morphological, cultural and biochemical characteristics (Collee and Marr, 1996).

\section{Antibiotic sensitivity test}

Antibiotic sensitivity test was performed by Kirby Bauer Disc Diffusion method (Bauer et al., 1966). Five different types of antibiotics were used in the study (Table 1). S. aureus strains were grown on nutrient agar at $37^{\circ} \mathrm{C}$ for 24 hours and the colonies were suspended in sterile saline water equivalent to a $0.5 \mathrm{McF}$ arland standard $(1.5 \mathrm{X} 108 \mathrm{CFU} / \mathrm{ml})$. Hisensitivity agar plate was uniformly seeded by adding $100 \mu 1$ inoculated broth and was spread by means of spreader. The discs were placed on each inoculated Hi-sensitivity agar plate. The plates were incubated at $37^{\circ} \mathrm{C}$ for 18 hours. The diameter of the zone of inhibition was observed in $\mathrm{mm}$ and the isolates were classified as "resistant" or "sensitive" based on the standard interpretative chart according to Clinical and Laboratory Standards Institute (CLSI) guidelines (CLSI, 2007).

\section{Antibacterial activity of rose petals extracts against $S$. aureus}

Antibacterial activity of Rose petals extracts was performed by well diffusion technique. 
S.aureus strains were grown overnight on nutrient agar at $37^{\circ} \mathrm{C}$, and the colonies were suspended in sterile saline water equivalent to a $0.5 \mathrm{McF}$ arland standard $(1.5 \times 108 \mathrm{CFU} / \mathrm{ml})$. The suspension $(100 \mu \mathrm{L})$ was spread over the Hi-Sensitivity agar. The wells of $6 \mathrm{~mm}$ diameter were cut into the agar medium with a sterilized cork borer. Then $20 \mu 1$ each of the extracts were added separately into the separate wells. The plates were incubated at $37^{\circ} \mathrm{C}$ for 18 hours. The diameter of the zone of inhibition around each well was measured and recorded (Bauer et al., 1966).

\section{Results and Discussion}

The present study was conducted to evaluate the effect of extracts of Rose petals on Staphylococcus aureus. It showed the comparative study of an extracts and antibiotic Ampicillin as a positive control and negative control (Methanol and Distilled water) against strains of S. aureus. Antibiotic susceptibility test showed that $100 \%$ S. aureus strains were found to be sensitive to
Erythromycin and Linezolid each, followed by $80 \%$ to Clindamycin and $60 \%$ to Gatifloxacin. However, all the strains of $S$. aureus were resistant to Oxacillin (Table 2). Antibacterial activity of Rose petals extracts showed that all S.aureus (100\%) were sensitive to both Methanol as well as Distilled Water extracts (Table 3 ).

Plant and plant materials have been widely used to cover health care needs, and this form of medicine is termed as herbal medicine, herbalism or herbology. Plant materials have been widely used for the treatment of infectious diseases and have gained popularity as they decrease the side effects seen with the use of systemic antimicrobials. Prior to using these plant and plant materials, a through screening of the plants should be done, which would help to isolate and characterize the active compounds. Rose and parts of the rose plant have been in use in medicine, the evidence for which dates back to the "Arkprakash" one of the vedas of Ayurveda (Bahl et al., 2016).

Table.1 Antibiotics discs used in the study

\begin{tabular}{|c|l|c|c|}
\hline Sr. No. & Antibiotics & Abbreviation & Concentration \\
\hline $\mathbf{1}$ & Clindamycin & $(\mathrm{CD})$ & $2 \mathrm{mcg}$ \\
\hline $\mathbf{2}$ & Erythromycin & $(\mathrm{E})$ & $15 \mathrm{mcg}$ \\
\hline $\mathbf{3}$ & Gatifloxacin & $(\mathrm{GF})$ & $10 \mathrm{mcg}$ \\
\hline $\mathbf{4}$ & Linezolid & $(\mathrm{LZ})$ & $10 \mathrm{mcg}$ \\
\hline $\mathbf{5}$ & Oxacillin & $(\mathrm{OX})$ & $5 \mathrm{mcg}$ \\
\hline
\end{tabular}

Table.2 Antibiotics susceptibility test of $S$. aureus

\begin{tabular}{|l|l|l|l|l|}
\hline Antibiotics & \multicolumn{2}{|c|}{ R } & \multicolumn{2}{|c|}{ S } \\
\hline Clindamycin & 1 & $20 \%$ & 4 & $80 \%$ \\
\hline & 0 & $0 \%$ & 5 & $100 \%$ \\
\hline Erythromycin & & & & \\
\hline Gatifloxacin & 2 & $40 \%$ & 3 & $60 \%$ \\
\hline Linezolid & 0 & $0 \%$ & 5 & $100 \%$ \\
\hline Oxacillin & 5 & $100 \%$ & 0 & $0 \%$ \\
\hline
\end{tabular}


Table.3 Antibacterial activity of rose petals extracts against $S$. aureus

\begin{tabular}{|c|c|c|c|c|c|}
\hline Extracts & Solvents & \multicolumn{2}{|c|}{ R } & \multicolumn{2}{|c|}{ S } \\
\hline \multirow{2}{*}{ Rose petals I } & Methanol & 0 & $0 \%$ & 5 & $100 \%$ \\
\hline \multirow{2}{*}{ Rose petals II } & D.W & 0 & $0 \%$ & 5 & $100 \%$ \\
& Methanol & 0 & $0 \%$ & 5 & $100 \%$ \\
\hline \multirow{2}{*}{ Negative control } & D.W & 0 & $0 \%$ & 5 & $100 \%$ \\
\hline & Methanol & 5 & $100 \%$ & 0 & $0 \%$ \\
\hline Positive control & Methanol & 5 & $100 \%$ & 0 & $0 \%$ \\
\hline (Ampicillin) & D.W & 1 & $0 \%$ & 5 & $100 \%$ \\
\hline
\end{tabular}

Rose petals I and II extracts were evaluated against five strains of skin infection causing $S$. aureus. Antibacterial activity of Rose petals extracts showed that all $S$. aureus $(100 \%)$ were sensitive to both Methanol as well as Distilled Water extracts Rose petals extracts possess moderate broad spectrum antimicrobial activity against gram positive, gram negative, acid fast bacteria and fungi. According to Aqueous extracts showed highly significant antibacterial activity while methanol extracts showed moderate activity (Vasanthakumar et al., 2015). In the study of Darokar et al., (1998), Rose petals extract were more effective against Gram positive bacteria than that of Gram negative bacteria. Owing to its fragrance, rose has been seen to influence culture artistically, economically, clinically and scientifically. This fragrance of rose is essentially due to oil secreted in the papillae, especially geraniol, citronellol, ethanol, rose oxide, linalool, nerol, eugenol (Sharma, 2003). Various parts of the rose plant, including the stem leaves and the petals have been observed to possess antibacterial and antifungal properties. These properties are noticed in the active compounds that are extracted from the plant, and the extraction of the same depends on the solvent that is used for the extraction process. The most commonly used solvents are methanol and ethanol. Alcohols are used as, they extract both polar and non-polar constituents from plants (Bisignio et al., 1999; Lourens et al., 2004).

In conclusion, the present study demonstrates antibacterial activity of Rose petals extracts against skin infection causing S.aureus. This study shows that rose petals may possess some chemical constituents which are responsible for antimicrobial activity that can partially minimize the use of antibiotics to some extent. However, further studies are required for screening of the volatile and nonvolatile active antimicrobial constituents of rose petals to utilize them in pharmaceutical industries.

\section{References}

Bahl D., Swati, A. Chakravarthy, S. Mutalik, and Devkar R. 2016. Determination of Antibacterial and Antifungal Properties of Rose Extract- An In vitro Study. International Journal of Pharmacognosy and Phytochemical Research. 8(10): 1695-1697.

Bauer, AW., W.M. Kirby, J.C. Sherris and Turck, M. 1966. Antibiotic Susceptibility testing by a standardized disk method. Am. J. Clin. Pathol. 45(4): 493-496.

Bisignino G., R. Sanogo, A. Marino, R. Aquino, V. D' angelo, M.P. Germano, R. De Pasquale and Pizza C. 1999. 
Antimicrobial activities of Mitracarpus scaber extract and isolated constituents. Lett. Appl. Microbiol. 30: 105 - 108.

Cantore P.L., V. Shanmugaiah and Lacobellis N.S. 2009. Antibacterial activity of essential oil components and their potential use in seed disinfection. J. Agric. Food Chem. 57: 9454-9461.

CLSI. 2007. Performance standards for antimicrobial susceptibility testing: 17 th Informational supplement, Approved standard M100-S17, Wayne, USA. Clinical and Laboratory Standards Institute.

Collee JG. and Marr W. 1996. Tests for identification of bacteria and laboratory control of antimicrobial therapy, Chapter 7 and chapter 8. In In: Mackie \& McCartney Practical Medical Microbiology, by Fraser A.G., Marmion B.P. and Simmons (Eds.) 14th ed. Collee J.G., 131-151. New York: Churchill Livingstone: New York.

Darokar, M.P., A. Mathur, S. Dwivedi and Bhalla, R. 1998. Detection of antibacterial activity in the floral petals of some higher plants. Curr. Sci. 75(3): 187.

Khan Jahir Alam and Tewari Saurabh 2011. A Study on Antibacterial Properties of Rosa indica against Various Pathogens. Asian Journal of Plant Science and Research. 1 (1): 22-30.

Kiruthika K. Anu, A. Amutha Jaisheeba and Sornara R. 2011. Evaluation of the antimicrobial Property of Selected Flower Extracts when exposed in a Hospital Environment. Int. J. Pharm. Tech. Res., 3(2).

Laxmi S.K., D.D. Sandhya, S. Geetha and
Lakshmi S.M. 2017. Biochemical and Antimicrobial Analysis of Rose Petals (Rosa indica). European journal of Pharmaceutical and Medical Research. 4(7): 637-640.

Lourens A.C.U., D. Reddy, K.H.C Baser., A.M. Viljoen and Van Vuuren S.F. 2004. In vitro biological activity and essential oil composition of four indigenous South African Helichrysum species. J Ethnopharmacol. 9: 253 - 258.

Özkan G., O. Sağdıç, N. Göktürk-Baydar and Baydar H. 2004. Antioxidant and antibacterial activities of Rosa damascene flower extracts. Food Sci Technol Int. 10: 277-281.

Saeed Rumana, Hameed-Ur-Rehman, Shaiq Ali, Hidayat Ullah, Mukhtar Ullah, Rohullah, Saeed Hassan, Farhan, Shehzad Ahmed and Akhwan Shomaila. 2014. Phytochemical Analysis and AntiMicrobial Activities of Rosa Indica Collected from Kohat Pakistan. AJPCT. 2 (12): 1370-1377.

Sahoo Anjulata Manjari, Chandra Kanti Chakraborti, Sarojini Nayak and Kayal Sriti 2011. Correlation between phytochemical screening and in vitro antibacterial activity study of Rosa indica linn. Leaves. IJRAP. 2 (5): 1595 $-1597$.

Sharma R. 2003. Medicinal plants of India. An Encyclopaedia. 215 - 217.

Vasanthakumar D., D. Aruna, M. Mujeebunisa, S. Saravanan and Divya V. 2015. Antibacterial activity of Rosa damascena petal extracts against the fish pathogen Aeromonas hydrophila. European Journal of Experimental Biology. 5(8):56-59.

\section{How to cite this article:}

Chandekar, C.J. and Pranita A. Gulhane. 2018. Inhibitory Potential of Rose Petals Extracts: An Attempt to Annihilate Staphylococcus aureus. Int.J.Curr.Microbiol.App.Sci. 7(12): 1555-1559. doi: https://doi.org/10.20546/ijcmas.2018.712.183 